\title{
Drug Development: Stages of Drug Development
}

Gamal Osman Elhassa*1 and Khalid Omer Alfarouk ${ }^{2}$

${ }^{1}$ Pharmaceutics Department, Faculty of Pharmacy,Omdruman Islamic University Omdruman ,Khartoum ,Sudan

${ }^{2}$ Alfarouk Biomedical Research LLC, San Antonio, Texas, USA

\begin{abstract}
New drug development program for the different compound is initiated because there is a disease or clinical condition without suitable pharmacotherapeutic products available. New drug development can proceed along varied pathways for different compounds. Drug invention programs result in the synthesis of compounds that are tested in assays and animal models.
\end{abstract}

Keywords: Drug development; Toxicology testing

\section{Introduction}

Drug development process involves rigorous testing and optimization of selected compounds to identify the drug that is mosteffective. This testing is done in cells (in vitro) and in animals (in vivo) to study the metabolism and to produce a product that is safe and has passed all regulatory requirements. Drugs failure in clinical practice is due in large part to two main reasons. The first reason is if they do not work properly and the second reason is, if they are not safe. The two most important issues to address in drug invention processes are; to identify a target and validation. This may be a protein receptor thatis associated with a disease condition, for this reason, it is important to know how the disease occurs at the molecular, cellular and genetic levels. Once the target is identified, then the next step is how the target plays a role in thedisease process. This is carried out by testing the target against different known and new compounds to know either one or several compounds that interact with the target and show either to neutralize or slow down the disease process.

\section{Stages of Drug Development}

\author{
$\triangleright$ Drug Invention \\ $\triangleright$ Product Characterization \\ $\triangleright$ Formulation\& development \\ $\triangleright$ Pharmacokinetics And Drug Disposition \\ $\triangleright$ Preclinical Toxicology Testing And IND Application \\ $\triangleright$ Bioanalytical Testing \\ $>$ Clinical Trials \\ $\triangleright$ Regulatory review \\ $\triangleright$ Marketing the product or drug \\ $\triangleright$ Post-marketing monitoring (phase-IV) trials
}

\section{Drug Invention}

Phenotypic screens as a renewed approach for drug discovery: Well before molecular target-based drug discovery became popular, phenotypic-based screening strategies were the foundation of pharmaceutical drug discovery. In the past 25 years, molecular targetbased drug screening has become the main drug discovery paradigm used in both the pharmaceutical industry and in academic translational research centres.
Specific delivery of biologicals: We have entered the era of biologicals. Although new chemical entities are still produced and successfully reach the market, many new biological products like antibodies and their derivatives, siRNA, cytokines, enzymes and other therapeutic peptides are now being developed. Already a third of all new therapeutic products in 2011 were biologicals rather than chemical derivatives.

The growing impact of click chemistry on drug discovery: Click chemistry is a modular approach that uses only the most practical and reliable chemical transformations. Its applications are increasingly found in all aspects of drug discovery, ranging from lead finding through combinatorial chemistry and targettemplated in situ chemistry, to proteomics and DNA research, using bio conjugation reactions. The copper-(I)-catalyzed 1, 2, 3-triazole formation from azides and terminal acetylenes is a particularly powerful linking reaction, due to its high degree of dependability, complete specificity, and the biocompatibility of the reactants. The triazole products are more than just passive linkers; they readily associate with biological targets, through hydrogen bonding and dipole interactions.

Targeting cancer-initiating cell drug resistance: a roadmap to a new generation of cancer therapies?: The occurrence of drug resistance in oncology accounts for treatment failure and relapse of diverse tumor types. Cancers contain cells at various stages of differentiation together with a limited number of 'cancer-initiating cells' able to self-renew and divide asymmetrically, driving tumorigenesis. Cancer initiating cells display a range of self-defense systems that include almost all mechanisms of drug resistance. Different molecular pathways and markers, identified in this malignant sub-population, are becoming targets for novel compounds and for monoclonal antibodies, which may be combined with conventional drugs. These interventions might eliminate drugresistant cancer-initiating cells and lead to remission or cure of cancer patients [1].

*Corresponding author: Gamal Osman Elhassan, Pharmaceutics Department Faculty of Pharmacy,Omdruman Islamic University Omdruman ,Khartoum ,Sudan , Tel: 966530965955; E-mail: gamaosma63@yahool.com

Received May 25, 2015; Accepted May 26, 2015; Published June 04, 2015

Citation: Elhassa GO, Alfarouk KO (2015) Stem Cell Therapy in Drug Discovery and Development. J Pharmacovigilance 3: e140. doi:10.4172/2329-6887.1000e141

Copyright: (c) 2015 Elhassa GO. This is an open-access article distributed under the terms of the Creative Commons Attribution License, which permits unrestricted use, distribution, and reproduction in any medium, provided the original author and source are credited. 
Biomarker-guided repurposing of chemotherapeutic drugs for cancer therapy: a novel strategy for drug development: This research strategy is commonly known as drug repurposing or drug repositioning and provides a faster path to the clinics. We have developed and implemented a modification of the standard drug repurposing strategy that we review here; rather than investigating target-promiscuous noncancer drugs for possible anti-cancer activity, we focus on the discovery of novel cancer indications for already approved chemotherapeutic anti-cancer drugs. Clinical implementation of this strategy is usually commenced at clinical phase II trials and includes pre-treated patients. As the response rates to any non-standard chemotherapeutic drug will be relatively low in such a patient cohort, it is a pre-requisite that such testing is based on predictive biomarkers. This review describes our strategy of biomarker-guided repurposing of chemotherapeutic drugs for cancer therapy, taking the repurposing of topoisomerase I (Top1) inhibitors and Top1 as a potential predictive biomarker as acase in point [2].

Oncoplastic surgery: Breast-conserving surgery (lumpectomy or partial mastectomy) can often be used for early-stage breast cancers. However, in some women, it can result in breasts of different sizes and/or shapes. For larger tumors, it might not even be possible, and a mastectomy might be needed instead. Some doctors address this problem by combining cancer surgery and plastic surgery techniques, known as oncoplastic surgery. This typically involves reshaping the breast at the time of the initial surgery, and may mean operating on the other breast as well to make them more symmetrical. This approach is still fairly new, and not all doctors are comfortable with it [3].

Scorpion Peptides: Potential Use for New Drug Development: Several peptides contained in scorpion fluids showed adiverse array of biological activities with high specificities to their targeted sites. Many investigations outlined their potent effects on microbes and showed their potential to modulate various biological mechanisms that are involved in immune, nervous, cardiovascular, and neoplastic diseases. Because of their essential structural and functional diversity, it is projected that scorpion-derived peptides could be used to develop new specific drugs [4].

Graphical abstract: The enormous progress in biotechnology, bioinformatics and nanotechnology made in recent years provides opportunities and scientific framework for thedevelopment of biomedicine and constitutes a paradigm shift in pharmaceutical R\&D and drug innovation. By analyzing the data and related information at $\mathrm{R} \& \mathrm{D}$ level over the past decades, developmental tendency and R\&D patterns were summarized [5] (Figure 1).

\section{Product Characterization}

When any new drug molecule shows a promising therapeutic

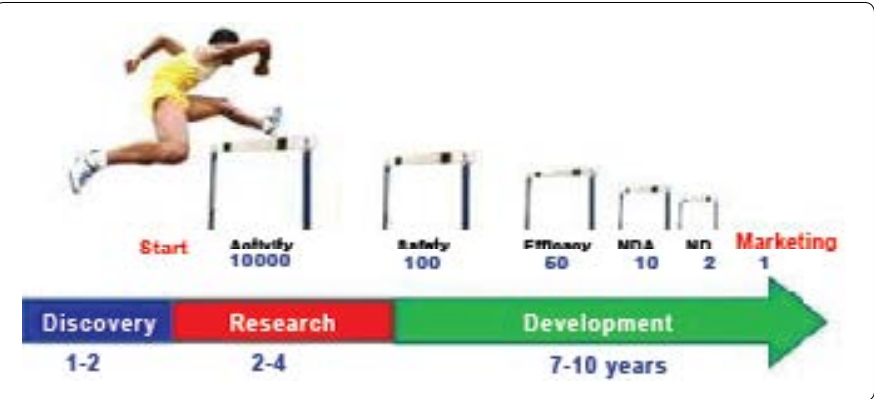

Figure 1: Graphical abstract. activity, then the molecule is characterized by its size, shape, strength, weakness, use, toxicity, and biological activity. Early stages of pharmacological studies are helpful to characterize the mechanism of action of the compound.

\section{Formulation and Development}

Pharmaceutical formulation is a stage of drug development during which the physicochemical properties of Active Pharmaceutical Ingredients (APIs) are characterized to produce a bioavailable, stable and optimal dosage form for a specific administration route [6].

During preformulation studies the following parameters are evaluated:

- Solubility in different media and solvents

- Dissolution of the active pharmaceutical ingredient (API)

- Accelerated Stability Services under various conditions

- Solid state properties (polymorphs, particle size, particle shape etc.)

- Formulation Services and Capabilities:

- Formulation development of new chemical entities (NCE)

- Optimization of existing formulations

- Process development for selected dosage forms

- Novel formulations for improved delivery of existing dosage forms

- Controlled release and sustained release formulations

- Self-emulsifying drug delivery systems

- Colloidal drug delivery systems

- Sub-micron and nano-emulsions

\section{Pharmacokinetics and Drug Disposition}

Pharmacokinetics studies provide useful data for formulation scientists. These studies provide data on parameters like $\mathrm{C}_{\max }, \mathrm{T}_{\max }$, A.U.C , etc.

\section{Preclinical Toxicology Testing and IND Application}

Toxicity testing for a new compound is very essential in drug development process. Toxicity of a substance can be observed by studying the accidental exposure to a substance, in vitro studies using cells, in vivo exposure to experimental animals. This preclinical toxicity testing is helpful to calculate no observed adverse effect level. Which are needed to initiate the clinical evaluation of investigational product [6] .The typical toxicology profile consists of safety pharmacology, genetic toxicology, acute and subchronic toxicology, absorption, distribution, metabolism, and excretion (ADME) studies, reproductive and developmental toxicity, and an evaluation of carcinogenic potential [7].

\section{Bioanalytical Testing}

BioanalyticalTesting supports most of the other activities related to drug development process. This work is used for proper characterization of thedrug molecule, developing optimal methods.

\section{Regulatory review}

After completion of clinical trials data obtained in clinical trials 
Citation: Elhassa GO, Alfarouk KO (2015) Drug Development: Stages of Drug Development J Pharmacovigilance 3: e141. doi:10.4172/2329$6887.1000 \mathrm{e} 141$

shows whether there is evidence to support the safety and efficacy of the drug in treating the disease condition. At this stage, the data collected is submitted to the relevant regulatory authorities to obtain approval to market the drug in their jurisdictions.

\section{Marketing the product}

The last stage in the drug development process is marketing of the product. Once it has been approved by regulatory authorities' here the drug manufacturers submit authorization application in each country or territory where they want to sell their product [8].

\section{Post-marketing monitoring (phase-IV) trials}

Post-marketing trials are used to assessthe safety of the drug in the marketplace. This may include reporting and investigation of the incidence and severity of rare adverse reactions, cost-effectiveness analyzes comparative trials, and quality of life studies [8].

\section{References}

1. www.drugdiscovery.com

2. Stenvang J, Kümler I, Nygård SB, Smith DH, Nielsen D (2013) Biomarkerguided repurposing of chemotherapeutic drugs for cancer therapy; a novel strategy in drug development. Front oncol 3: 313.

3. www.cancer.org/cancer/breastcancer/detaile dguide.

4. Hmed NB, Serria HT and Mounir ZK (2013) Scorpionpeptides: Potential use for new drug development, Journal of Toxicology 2013.

5. Single-dose (Acute) and pilot (DRF) toxicity testing in drug safety evaluation (2010) Drug safety evaluation, 2nd edition.

6. Parasuraman S (2011) Toxicological screening. J PharmacolPharmacother 2:74-79.

7. Dorato MA, Buckley LA (2007) Toxicology testing in drug discovery and development, current protocols in toxicology.

8. Dunne S, Shannon B, Dunne C, Cullen W (2013) A Review of the differences and similarities between generic drugs and their originator counterparts, including economic benefits associated with usage of generic medicines using Ireland as a case study. BMC Pharmacology and Toxicology 14:1. 